\title{
Quantization Principles Based on the Shielding Effect and Planck's Constant in Gravitational Fields
}

\author{
Hua Ma \\ College of Science, Air Force University of Engineering, Xi'an, People's Republic of China \\ Email address: \\ mahuar@xjtu.edu.cn \\ To cite this article: \\ Hua Ma. Quantization Principles Based on the Shielding Effect and Planck's Constant in Gravitational Fields. American Journal of Modern \\ Physics. Vol. 7, No. 4, 2018, pp. 131-135. doi: 10.11648/j.ajmp.20180704.12
}

Received: July 2, 2018; Accepted: July 11, 2018; Published: August 2, 2018

\begin{abstract}
To reveal the physical nature of Planck's constant, an analytic expression of Planck's constant is presented, and based on this expression, the De Broglie's relation and the expression of momentum operator are derived. To calculate Planck's constant, the shielding effect of the fundamental interaction is introduced, and found that Planck's constant can be calculated for the fundamental interaction fields with shielding effects, thus have obtained the general quantization principle: the systems with shielding effects can be quantized. As a result, the representation of Planck's constant in the gravitational field is derived, indicating that although the gravitational field can't be quantified, which has effects on quantum phenomena through expressing Planck's constant based on the curvature of space-time. This work is of significance for deepening the understanding of quantum mechanics, and for exploring the quantum mechanism in the cosmic celestial bodies.
\end{abstract}

Keywords: Quantum Mechanics, Planck’s Constant, De Broglie's Relation, Momentum Operator, Fundamental Interaction, Shielding Effect, Quantization Principle, Gravitational Field

\section{Introduction}

Quantum mechanics is a basic theory in physics. It describes the minimum scale properties of atoms and subatomic particles. The difference between quantum mechanics and classical physics is that the energy, momentum and other quantities of systems can be restricted to discrete values and objects [1-4]. Quantum mechanics originated from Planck's theoretical explanation of blackbody radiation phenomenon and was gradually improved. It has experienced many milestones, including the assumption of Planck's and Einstein's energy quantization, the establishment of Schrodinger's equation, the proposal of De Broglie's relation, the establishment of Heisenberg's uncertain principle and so on. Although quantum mechanics has entered a perfect stage, there are still some unsolved theoretical suspense, such as: why can energy be quantized? What is the theoretical basis of De Broglie's relation? What is the physical property of Planck's constant? Why can't the gravitational field be quantized? How can Planck's constant in the gravitational field be expressed? How to deduce the expression of the momentum operator?

The author has thought over these problems for many years, and finally found a way to understand Planck's constant. Based on this method, the analytical expression of Planck's constant is proposed, and some quantization principles are explained. The work is reported in this article. First, the analytical expression of Planck's constant is given. On this basis, the expressions of De Broglie's relation and momentum operator are derived, which are in agreement with the existing theories and experiments, and can be extended to a more general form. Secondly, the shielding effect of fundamental interactions is introduced to calculate Planck's constant and to explain the quantization principles. It is found that whether the force fields can be quantized depends on whether the fundamental interaction has shielding effects in the occupied area of the topological charges. This conclusion reasonably explains the problem that the gravitational field can't be quantized. Thirdly, the expression of Planck's constant in the gravitational field has been derived, indicating that although the gravitational field can't be quantized, which has effects on quantum phenomena through expressing Planck's constant based on the curvature of space-time.

In the following derivations and discussions, the space-time dimension defaults to 4 , and the vectors are all 4-dimensional. Thus, the 4-dimensional position vector, momentum, wave 
vector and velocity are $X^{v}=\left(c t, x^{j}\right), P^{\mu}=\left(E / c, p^{j}\right), K^{\mu}=$ $\left(\omega / c, k^{j}\right)$ and $U^{\mu}=\left(c, u^{j}\right)$, respectively. Where, $c$ is vacuum light speed, $\omega$ is angular frequency, $E$ is energy, $t$ is time, $\mu, v=0,1,2,3$, and $j=1,2,3$. Note that, use $g_{\mu v}$ as the space-time metric tensor, and establish it by orthogonal processing the time axis, so in this article adopting the orthogonal system of time axis is default [5], namely: $g_{00}>0, g_{0 j}=0$.

\section{An analytic Expression of Planck's Constant}

According to the field theory [6-9], when a topological charge insert the field produced by the same topological charge, the related force will be produced. Generally, the fundamental interaction force is proportional to the amount of topological charges and the field strength, so the value of the force is equal to the product of the amount of topological charges and the field strength. Taking account of these two factors, a fundamental interaction force with harmonic transfer form can be represented uniformly through the following formula:

$$
\begin{aligned}
& F^{\mu}(\theta)=\mathrm{A}^{\mu} e^{-\mathrm{i} \theta} \\
& F^{\mu}(\theta+\Delta \theta)=\mathrm{A}^{\mu} e^{-\mathrm{i}(\theta+\Delta \theta)}=\mathrm{A}^{\mu} e^{-\mathrm{i} \theta} e^{-\mathrm{i} \Delta \theta} \cong \mathrm{A}^{\mu} e^{-\mathrm{i} \theta}(1-\mathrm{i} \Delta \theta)
\end{aligned}
$$

Therefore the force change is

$\Delta F^{\mu}=F^{\mu}(\theta+\Delta \theta)-F^{\mu}(\theta)=-\mathrm{i} \Delta \theta \mathrm{A}^{\mu} e^{-\mathrm{i} \theta}=-\mathrm{i} F^{\mu} \Delta \theta$

Eq. (5) indicates that phase change $\Delta \theta$ brings to the force change $-\mathrm{i} F^{\mu} \Delta \theta$, where $-\mathrm{i}$ denotes that there are difference

$$
w=g_{\mu \nu} F^{\mu} \Delta \theta \Delta X^{v}=g_{\mu \nu} F^{\mu} \Delta t \Delta X^{v} \frac{\Delta \theta}{\Delta t}=g_{\mu \nu} P^{\mu} \Delta X^{v}\left(\frac{g_{00} \omega \Delta t+g_{j j} k^{j} \Delta x^{j}}{\Delta t}\right)
$$

In Eq. (6), $g_{\mu \nu} P^{\mu} \Delta X^{v}$ has the dimension of action. Here, limit this focus on single quantum field, and introduce a new physical quantity $\hbar$ :

$$
\hbar \equiv g_{\mu \nu} P^{\mu} \Delta X^{v}
$$

Historically, $\hbar$ was used to represent Planck's constant. Here using it to denote $g_{\mu \nu} P^{\mu} \Delta X^{v}$ of single quantum field. Whether $g_{\mu \nu} P^{\mu} \Delta X^{v}$ has the meaning of Planck's constant? In the next section, by deducing De Broglie's relation and momentum operator expression, this question is answered, and come to a conclusion that Eq. (7) is the analytic expression of Planck's constant for single quantum field.

\section{Derivation of De Broglie's Relation and the Momentum Operator Expression}

De Broglie's relation and momentum operator expression are the basis of quantum mechanics $[1,2,4]$, which are established by conjectures and analogies, and there are no theoretical derivation processes at present. Now, the upper definition of Planck's constant is adopted to deduce the De Broglie's relation and momentum operator expression, so as to verify the correctness of the definition and solve the problem about the basic principles of quantum mechanics.

Firstly, deduce De Broglie's relation. Taking $u^{j}=\Delta x^{j} / \Delta t$ and $\hbar$ into Eq. (6), gets:

$$
w=\hbar\left(g_{00} \omega+g_{j j} k^{j} u^{j}\right)=g_{00} \hbar \omega+g_{j j} \hbar k^{j} u^{j}
$$

According to the definition of power, power is equal to the product of momentum and time, namely:

$$
w=g_{\mu \nu} P^{\mu} U^{v}=g_{00} \frac{E}{c} c+g_{j j} p^{j} u^{j}=g_{00} E+g_{j j} p^{j} u^{j}
$$

Physically, the power in formulas (8) and (9) are equal, thus can obtain the following relation by comparing these two equations:

$$
E=\hbar \omega, p^{j}=\hbar k^{j}
$$

It is clear that, Eq. (10) is just the De Broglie's relation, proving that $\hbar$ has the physical meaning of Planck's constant. Further, this relation can be extended to 4-dimensional De 
Broglie's relation with more general covariant form:

$$
P^{\mu}=\hbar K^{\mu}
$$

Then, deduce the momentum operator expression. Force $F^{\mu}(\theta)$ is the function of phase $\theta$, and phase $\theta$ is the function of space-time position $X$, so the force $F^{\mu}(\theta)$ is the function of $X$, and can be expressed as follows:

$$
F^{\mu}=F^{\mu}(\theta(X))=\varphi^{\mu}(X)
$$

Note that, in the following derivations of this section, Einstein's summation rule does not be used for the coordinate index $v$ in Eq. (13)-(17). When the space-time position moves $\Delta X^{v}$ slightly, force $\varphi^{\mu}(X)$ will change to $\varphi^{\prime \mu}=$ $\varphi^{\mu}\left(X+\Delta X^{v}\right)$. The Taylor expansion of the force is

$$
\begin{aligned}
\varphi^{\prime \mu} & =\varphi^{\mu}\left(X+\Delta X^{v}\right)=\varphi^{\mu}(X)+\left(\Delta X^{v}\right) \partial_{v} \varphi^{\mu}(X)+\frac{\left(\Delta X^{v}\right)^{2}}{2 !} \partial_{v}^{2} \varphi^{\mu}(X)+\cdots \\
& =\left[1+\left(\Delta X^{v}\right) \partial_{v}+\frac{\left(\Delta X^{v}\right)^{2}}{2 !} \partial_{v}^{2}+\cdots\right] \varphi^{\mu}(X)=e^{\Delta X^{v} \partial_{v}} \varphi^{\mu}(X)
\end{aligned}
$$

The change of phase $\theta$ caused by the position change $\Delta X^{v}$ is $\theta \rightarrow \theta+\Delta \theta$, and thus the force $F^{\mu}$ will become to $F^{\prime \mu}=F^{\mu}(\theta+\Delta \theta)$ :

$$
F^{\prime \mu}=F^{\mu}(\theta+\Delta \theta)=e^{-\mathrm{i} \Delta \theta} F^{\mu}(\theta)
$$

According to Eq. (12), $F^{\mu}(\theta+\Delta \theta)=\varphi^{\mu}\left(X+\Delta X^{v}\right)$, and $F^{\mu}(\theta)=\varphi^{\mu}(X)$, using these results to Eq. (13) and (14), comes to:

$$
e^{\Delta X^{v} \partial_{\nu}} \varphi^{\mu}(X)=e^{-\mathrm{i} \Delta \theta} \varphi^{\mu}(X)
$$

Obviously, Eq. (15) presents that, operator $e^{\Delta X^{v} \partial_{v}}$ act on wave function $\varphi^{\mu}(X)$ in the left term, whereas physical quantity $e^{-\mathrm{i} \Delta \theta}$ is multiplied by wave function $\varphi^{\mu}(X)$ in the right term. According to the operator theory, $e^{\Delta X^{v} \partial_{v}}$ is the operator of $e^{-\mathrm{i} \Delta \theta}$ when they act on the same function with the same result, so:

$$
\Delta X^{v} \partial_{v} \leftrightarrow-\mathrm{i} \Delta \theta=-\mathrm{i} g_{v v} K^{v} \Delta X^{v}
$$

Using the principle of operator correspondence to solve relation (16), the operator $\widehat{K}^{v}$ of wave vector $K^{v}$ can be derived as:

$$
\widehat{K}^{v}=\frac{-\Delta X^{v} \partial_{v}}{\mathrm{i} g_{v v} \Delta X^{v}}=\mathrm{i} \frac{\partial_{v}}{g_{v v}}
$$

Popularizing the above expression to general metric of space-time, operator $\widehat{K}^{\mu}$ is

$$
\widehat{K}^{\mu} \equiv \mathrm{i} g^{\mu \nu} \frac{\partial}{\partial x^{v}}
$$

Considering the conclusion of Eq. (11), the expression of momentum operator is

$$
\widehat{P}^{\mu} \equiv \hbar \widehat{K}^{\mu}=\mathrm{i} \hbar g^{\mu \nu} \frac{\partial}{\partial x^{v}}
$$

As an example of verification, using this expression to flat space-time with $g_{00}=1$ and $g_{j j}=-1$, can obtain $\hat{P}^{0}=$ $\mathrm{i} \hbar \mathrm{c}^{-1} \partial / \partial t$, and the energy and momentum operators in flat space-time are:

$$
\widehat{H}=c \hat{P}^{0}=\mathrm{i} \hbar \frac{\partial}{\partial t}, \hat{P}^{j}=\mathrm{i} \hbar \frac{\partial_{j}}{g_{j j}}=-\mathrm{i} \hbar \frac{\partial}{\partial x^{j}}
$$

By now, based on the expression of $\hbar$ with Eq. (7), De Broglie's relation and momentum operator expression are deduced, and the conclusion are consistent with the existing theories. It not only prove the correctness of $\hbar$ expression, but also solve the suspense of De Broglie's relation and the momentum operator expression.

\section{Quantization Principle Based on the Shielding Effect}

According to the definition of Eq. (7) and De Broglie's relation of Eq. (10), the effect of $\hbar$ is equivalent to Planck's constant in quantum mechanics. Exploring a method to calculate $\hbar$ based on Eq. (7) and to prove it to be a constant, helps to provide a reasonable physical explanation on Planck's constant, and to deepen understanding of the quantization principle. To resolve this problem, the theoretical derivation should begin with the discussions of the fundamental interaction force properties.

In the orthonormal system of time axis, Eq. (7) can be simplified as:

$$
\hbar=g_{\mu \nu} P^{\mu} \Delta X^{v}=g_{00} E \Delta t+g_{j j} p^{j} \Delta x^{j}
$$

which can be used as the general expression to calculate $\hbar$. When $\hbar$ is regarded as Planck's constant, the expression of Eq. (1) should be limited as single quantum field, and the calculation area should be selected sensibly to guarantee that the result is constant. Tracing back to the expression of Eq. (6) and (7), $P^{\mu}$ is the momentum of quantum field, $\Delta X^{v}$ is the space-time size of a single quantum within which the force field do works. So the basic criterion for quantizing force field is that: under what conditions can the minimum action value calculated from Eq. (21) be constant?

To answer this question, here I introduce the shielding effect of the fundamental interactions [11, 12]. From the field theory, the four fundamental interactions are all originated from the field sources. These field sources can also be called topological charges, and some have shielding effects. The shielding effect is a basic physical phenomenon associated with the fundamental interactions, namely when the topological charge is placed in the fundamental interaction fields, the field force lines in the area occupied by the topological charge will be interrupted, resulting in that the internal force field of the shielded region becomes to zero. Currently, there is no scientific explanation for the physical principle of the shielding effect, and not all fundamental interactions have this effect, but this phenomenon is verified 
by experiments and is accepted by the physics world. Here, the shielding effect is introduced as the basic principle to discuss the quantization problem, and to solve the analytical calculation of Planck's constant.

For gravitational interaction force, there is no shielding effect in the fields, and thus the action scope overlay all the space, as shown by Figure 1(a). In the regions occupied by mass elements which are regarded as the topological charges of gravitational fields, the total gravitational fields are composed by the fields from other external mass and test mass element itself. Especially in the region with small size of $\Delta X^{v} \rightarrow 0$, the magnitude of gravitational field varies drastically according to Newton's law of universal gravitation and Einstein's theory of general relativity $[13,14]$. So, for gravitational interaction force, its energy $E$ and momentum $p^{j}$ have great changes in regions occupied by topological mass charges, and thus $\hbar$ expressed by Eq. (21) is not constant at small space-time with size of $\Delta X^{v} \rightarrow 0$. It comes to a conclusion that the gravitational field can't be quantized.
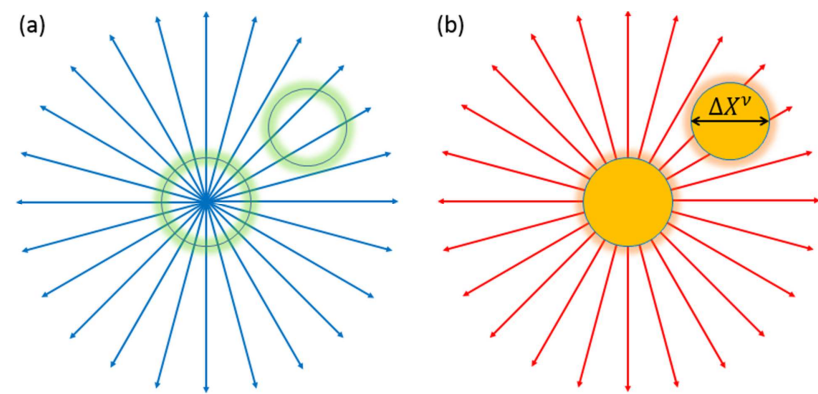

Figure 1. The shielding model of physical field in the region occupied by source charge: (a) no shielding effect for the gravitational interaction force, (b) there are shielding effects for the non-gravitational interaction forces.

The second kinds of fundamental interaction forces are non-gravitational interaction forces, such as electromagnetic interaction force, strong interaction force and weak interaction force. Different from the gravitational field, these three force fields all have shielding effects, and can be unified by the gauge field theory [7]. As shown by Figure 1(b), in the internal region occupied by topological charges, the interaction force is zero, namely $f^{j}=0$ and $p^{j}=0$. In the shielded area, using the result of $p^{j}=0$ to Eq. (21), the value of $\hbar$ for these non-gravitational interaction forces is

$$
\hbar=g_{00} E \Delta t
$$

Physically, Planck's constant represents the minimum action of elementary particles. For the non-gravitational fields with shielding effects, the action of their elementary topological charges can be calculated by Eq. (22), indicating that the value is determined by energy and time. So, to calculate $\hbar$, the action area should be limited in the shielded area, and $\Delta X^{v}$ are regarded as the size of the shielded area. According to the energy conservation principle, the energy $E$ in the shielded area keeps constant for that the force in it is zero. According to the relativistic effect in the shielded area with size of $\Delta X^{v} \rightarrow 0$, the field velocity is light speed, and the region is immobile, so the space-time interval is $(\Delta s)^{2}=$ $g_{\mu \mu}\left(\Delta X^{\mu}\right)^{2}=0$, which limits that the time change $\Delta t$ is

$$
\Delta t=\frac{1}{c} \sqrt{\frac{-g_{j j}\left(\Delta x^{j}\right)^{2}}{g_{00}}}
$$

For the non-gravitational interaction forces, the size of their source charges is constant, resulting in that the size $\Delta x^{j}$ of the shielded area is constant. Using this conclusion to Eq. (23), comes to a result that the time change $\Delta t$ is also constant. Obviously, in the shielded region, the energy and the time change are all constant, so the Planck's constant determined by Eq. (22) is a constant, indicating that the non-gravitational fundamental interaction force fields can be quantized.

From the definition of Planck's constant and the above discussions about the shielding effect, a conclusion comes to that, the fundamental interaction fields with shielding effects can be quantized, and Planck's constant can be analytically expressed and calculated. The conclusion can also be extended to the general quantization principle, that is, the systems with shielding effects can be quantized.

\section{Planck's Constant in the Gravitational Field}

The gravitational field itself can't be quantized, so there is no corresponding Planck's constant. The other three kinds of fundamental interaction forces can be quantized, and the corresponding Planck's constant can be calculated by Eq. (22). According to the theory of gravitational geometry, the gravitational field will bring to the space curvature, so Planck's constant expressed by Eq. (22) will reflect the curvature effect in the gravitational field.

Firstly, considering the flat space with $g_{00}=1$, and supposing that $E=E_{0}, \Delta t=\Delta \tau$, the value of $\hbar$ from Eq. (22) is

$$
\hbar=g_{00} E \Delta t=E_{0} \Delta \tau \equiv \hbar_{0}
$$

here $\hbar_{0}$ is used to denote the Planck's constant in the flat space, and in fact, can be understood as the traditional Planck's constant.

Next consider the non-flat space caused by the gravitational field. According to the general relativity theory, the energy $E_{0}$ and time $\Delta \tau$ in the flat space will change to $E$ and $\Delta t$ in the non-flat space, and the values can be calculated using space-time metric $[9,14]$ :

$$
E=E_{0} \sqrt{g_{00}}, \Delta t=\Delta \tau / \sqrt{g_{00}}
$$

Taking Eq. (25) into Eq. (22), and adopting the result of Eq. (24), Planck's constant in the gravitational field can be obtained:

$\hbar=g_{00} E \Delta t=g_{00}\left(E_{0} \sqrt{g_{00}}\right) \frac{\Delta \tau}{\sqrt{g_{00}}}=g_{00} E_{0} \Delta \tau=g_{00} \hbar_{0}$

The upper formula indicates that Planck's constant is related to the space curvature. The traditional Planck's constant is obtained through measurements, whether it is the function of space curvature has not been revealed. In the universe, our space is almost flat compared with black holes, 
so the influence of space curvature on Planck's constant can be ignored in our space, but it should be taken full consideration in black hole physics. Eq. (26) offers theoretical predictions of how the space curvature influence on quantum phenomena, and verifies the correctness of the gravitational geometric effect, providing a theoretical way to explore the quantum effects in black holes $[15,16]$.

\section{Conclusion}

To reveal the physical nature of Planck's constant, an analytic expression of Planck's constant is presented, and based on this expression, the De Broglie's relation and the expression of momentum operator are derived. The theoretical results are in good agreement with the existing theories and experiments, which proves the correctness of the analytical expression and provides a theoretical basis for the establishment of De Broglie's relation and momentum operator expression. To calculate Planck's constant, the shielding effect of the fundamental interaction is introduced, found that Planck's constant can be calculated for the fundamental interaction field with shielding effects, and thus the fields can be quantized. Conversely, Planck's constant can't be calculated for the fundamental interaction field without shielding effects, so the field can't be quantized. This conclusion is reflected in the four fundamental interaction fields, and also reveal why the gravitational field can't be quantized, clarifying the basic principle of quantization. As a result, the representation of Planck's constant in the gravitational field is derived, showing that Planck's constant is a function of space-time curvature, so the variation of curvature caused by gravitational fields will affect quantum phenomena.

Among the ideas proposed in this paper, only the analytic expression of Planck's constant and the shielding effect have some conjecture, but the conclusions are consistent with the existing theories and experiments, proving that these ideas are reasonable. In order to further verify the rationality of this work, some theoretical improvements and extensions need to make. For instance, the force field model with shielding effects should be extended to a system with shielding effects, and thus propose the general quantizing principle that systems with shielding effects can be quantized. The expression of Planck's constant in the gravitational field should be applied to the black hole physics, using which to predict the quantum phenomena in celestial bodies. The work in this paper is of significance for deepening the understanding of quantum mechanics, and needs further improvements.

\section{Acknowledgements}

The author is grateful to the support from the National Natural Science Foundation of China (Grant No. 61331005), and the Innovation Team of Shaanxi province (Grant No. 2014KCT-05).

\section{References}

[1] Griffiths, David J., Introduction to Quantum Mechanics (Prentice Hall, 2nd ed., 2004).

[2] J. J. Sakurai, Modern Quantum Mechanics (Massachusetts: Addison-Wesley, 1995).

[3] T. Hey, P. Walters, The New Quantum Universe (Cambridge University Press, 2009).

[4] D. McMahon, Quantum Mechanics Demystified (Mc Graw Hill (USA), 2006).

[5] Shimin Wu, General relativity theory (Beijing Normal University press (in Chinese), 1998).

[6] F. Mandl, G. Shaw, Quantum Field Theory (John Wiley \& Sons. 1993).

[7] Frampton, Gauge Field Theories (Wiley, Frontiers in Physics (2nd ed.), 2000).

[8] I. T. Adamson, Introduction to Field Theory (Dover Publications, 2007).

[9] Qinren Zhang, Classical field theory (Beijing: Science Press (in Chinese), 2003).

[10] Hua Ma, A Physical Explanation on Why Our Space Is Three Dimensional, American Journal of Modern Physics. Vol. 6, No. 6, 2017.

[11] Davies, Paul, The Forces of Nature (Cambridge Univ. Press (2nd ed.), 1986).

[12] Weinberg, Steven, Dreams of a Final Theory (Basic Books, 1994).

[13] Callahan, J. James, The Geometry of Space-time: an Introduction to Special and General Relativity (New York: Springer, 2000).

[14] Liao Liu, General relativity theory (Beijing: Higher Education Press (in Chinese), 1987).

[15] Ta-Pei Cheng, Relativity, Gravitation and Cosmology: A Basic Introduction (Oxford and New York: Oxford University Press, 2005).

[16] M. Robert Wald, Space, Time, and Gravity: the Theory of the Big Bang and Black Holes (Chicago: University of Chicago Press, 1992). 